\title{
An Effective Bicubic Convolution Interpolation-Based Iterative Luma Optimization for Enhancing Quality in Chroma Subsampling
}

This paper was downloaded from TechRxiv (https://www.techrxiv.org).

\section{LICENSE}

CC BY 4.0

SUBMISSION DATE / POSTED DATE

$31-08-2021 / 10-09-2021$

\section{CITATION}

Chung, Kuo-Liang; Huang, Chih-Yuan; Kao, Chen-Wei (2021): An Effective Bicubic Convolution InterpolationBased Iterative Luma Optimization for Enhancing Quality in Chroma Subsampling. TechRxiv. Preprint. https://doi.org/10.36227/techrxiv.16544898.v1

$\mathrm{DOI}$ 


\title{
An Effective Bicubic Convolution Interpolation-Based Iterative Luma Optimization for Enhancing Quality in Chroma Subsampling
}

\author{
KUO-LIANG CHUNG ${ }^{1}$, (Senior Member, IEEE), CHIH-YUAN HUANG ${ }^{1}$, AND CHEN-WEI \\ $\mathrm{KAO}^{1}$ \\ ${ }^{1}$ Department of Computer Science and Information Engineering, National Taiwan University of Science and Technology, Taipei 10672, Taiwan \\ Corresponding author: Kuo-Liang Chung (e-mail: klchung01@gmail.com).
}

This work was supported by the contracts MOST-108-2221-E-011-077- MY3 and MOST-110-2221-E-011-088-MY3 of the Ministry of Science and Technology, Taiwan.

\begin{abstract}
Traditionally, prior to compressing an RGB full-color image, for each converted $2 \times 2$ $\mathrm{CbCr}$ block $B^{C b C r}$, chroma subsampling only downsamples $B^{C b C r}$, but without changing the luma block $B^{Y}$ at all. In the current research, a special linear interpolation-based, namely the COPY-based, chroma subsampling-first luma modification (CSFLM) study has attempted to change the luma block for enhancing the quality of the reconstructed RGB full-color image. In this paper, a fast and effective nonlinear interpolation, namely the bicubic convolution interpolation (BCI), based iterative luma modification method for CSFLM is proposed. In our iterative method, a BCI-based distortion function and its convex property proof are first provided. Next, based on the proposed convex distortion function, a pseudoinverse technique is applied to obtain the initial luma modification solution, and then an iterative method is proposed to improve the initial luma modification solution. Based on five testing image datasets, namely the IMAX, Kodak, SCI (screen content images), CI (classical images), and Video datasets, the thorough experimental results have demonstrated that on the newly released Versatile Video Coding (VVC) platform VTM-12.0, our iterative luma modification method achieves substantial quality, execution-time, and quality-bitrate tradeoff improvements when compared with the existing state-of-the-art methods.
\end{abstract}

INDEX TERMS Chroma subsampling-first luma modification (CSFLM), Convex distortion function, Iterative luma optimization, Quality-bitrate tradeoff, Quality enhancement, RGB full-color image, Versatile video coding (VVC).

\section{INTRODUCTION}

DIOR to compression, the input RGB full-color image $I^{R G B}$ is first converted to a YCbCr image $I^{Y C b C r}$ using the BT.601-5 transformation [8]:

$$
\left[\begin{array}{c}
Y_{i} \\
C b_{i} \\
C r_{i}
\end{array}\right]=\left[\begin{array}{ccc}
0.257 & 0.504 & 0.098 \\
-0.148 & -0.291 & 0.439 \\
0.439 & -0.368 & -0.071
\end{array}\right]\left[\begin{array}{c}
R_{i} \\
G_{i} \\
B_{i}
\end{array}\right]+\left[\begin{array}{c}
16 \\
128 \\
128
\end{array}\right]
$$

where $\left(Y_{i}, C b_{i}, C r_{i}\right), 1 \leq i \leq 4$, denotes the $i$ th YCbCr triple-value of each $2 \times 2 \mathrm{YCbCr}$ block $B^{Y C b C r}$, and $\left(R_{i}, G_{i}, B_{i}\right)$ denotes the $i$ th triple-value of the collocated $2 \times 2$ ground truth RGB full-color block $B^{R G B}$.

Conventionally, chroma subsampling only downsamples each $2 \times 2 \mathrm{CbCr}$ block $B^{C b C r}$, but without changing the collocated luma block $B^{Y}$ at all. There are two chroma subsampling formats, namely 4:2:2 and 4:2:0. 4:2:0 downsamples the $(C b, C r)$-pair for each $B^{C b C r} ; 4: 2: 2$ downsamples the $(\mathrm{Cb}, \mathrm{Cr})$-pair for each row of $B^{C b C r}$.

In the chroma subsampling-first luma modification (CSFLM) scheme [4], as depicted in Fig. 1], after performing the chroma subsampling method, such as 4:2:0(A), 4:2:0(L), 4:2:0(R), 4:2:0(DIRECT), or Anchor [12], on $B^{C b C r}$, it attempts to modify each luma value in $B^{\bar{Y}}$ for better enhancing the quality of the reconstructed RGB full-color image. 


\section{A. RELATED CHROMA SUBSAMPLING WORKS}

In this subsection, we briefly introduce the above-mentioned five widely used chroma subsampling methods.

4:2:0(A) calculates the subsampled $(\mathrm{Cb}, \mathrm{Cr})$-pair by averaging the four chroma pairs of $B^{C b C r} .4: 2: 0(\mathrm{~L})$ and 4:2:0(R) calculate their subsampled $(\mathrm{Cb}, \mathrm{Cr})$-pairs by averaging the chroma pairs in the left and right columns of $B^{C b C r}$, respectively. 4:2:0(DIRECT), which is abbreviated as 4:2:0(D), takes the top-left chroma pair of $\mathrm{B}^{\mathrm{CbCr}}$ as the subsampled $(\mathrm{Cb}, \mathrm{Cr})$-pair. Throughout this paper, the term " $(\mathrm{Cb}, \mathrm{Cr})$-pair" and the term "chroma pair" denote the same thing.

The Anchor method [12] calculates the subsampled chroma pair by first performs a 3-tap filter $[1,6,1] / 8$ at the leftmost location of each row of $B^{C b C r}$, and then performs a 3-tap filter $([0,4,4] / 8)^{T}$ at the top-left entry of $B^{C b C r}$ which has been updated by the first step.

\section{B. RELATED CSFLM WORKS}

At the server side of Fig. 1, as a preprocessing step of the CSFLM scheme [4], chroma subsampling is first performed on $B^{C b C r}$ to obtain the subsampled $(\mathrm{Cb}, \mathrm{Cr})$-pair, namely $\left(C b_{s}, C r_{s}\right)$.

Next, a COPY-based upsampling process is applied to duplicate the chroma pair $\left(\mathrm{Cb}_{s}, \mathrm{Cr}_{s}\right)$ four times for constructing an estimated $2 \times 2 \mathrm{CbCr}$ block $B^{e s t, C b C r}$, where each entry $\left(C b_{i}^{e s t}, C r_{i}^{e s t}\right), 1 \leq i \leq 4$, equals $\left(C b_{s}, C r_{s}\right)$. Here, the COPY-based upsampling method is exactly the nearest neighbor (NN) based upsampling method provided by the Versatile Video Coding (VVC) standard [18] and its predecessor High Efficiency Video Coding (HEVC) standard [6].

Using the $i$ th estimated $(\mathrm{Cb}, \mathrm{Cr})$-pair of $B^{e s t, C b C r}$, namely $\left(C b_{i}^{e s t}, C r_{i}^{e s t}\right), 1 \leq i \leq 4$, and the luma modification parameter, namely $Y_{i}^{\prime}$, the $i$ th RGB full-color pixel-distortion function $P D\left(Y_{i}^{\prime}\right)$ is delivered to measure the sum of square errors (SSE) between the $i$ th ground truth RGB full-color pixel $\left(R_{i}, G_{i}, B_{i}\right)$ and the $i$ th estimated RGB full-color pixel $\left(R_{i}^{e s t}, G_{i}^{e s t}, B_{i}^{e s t}\right)$. $\left(R_{i}^{e s t}, G_{i}^{e s t}, B_{i}^{e s t}\right)$ can be obtained by replacing $C b_{i}$ and $C r_{i}$ in Eq. (2) with $C b_{s}$ and $C r_{s}$, respectively:

$$
\left[\begin{array}{c}
R_{i} \\
G_{i} \\
B_{i}
\end{array}\right]=\left[\begin{array}{ccc}
1.164 & 0 & 1.596 \\
1.164 & -0.391 & -0.813 \\
1.164 & 2.018 & 0
\end{array}\right]\left[\begin{array}{c}
Y_{i}-16 \\
C b_{i}-128 \\
C r_{i}-128
\end{array}\right]
$$

Setting the estimated $i$ th RGB full-color pixel $\left(R_{i}^{e s t}\right.$, $\left.G_{i}^{e s t}, B_{i}^{e s t}\right)$ to equal the ground truth $i$ th full-color pixel $\left(R_{i}, G_{i}, B_{i}\right)$, it yields an equation for each color $\in$ $\{R, G, B\}$, resulting in the following three equations:

$$
\begin{aligned}
Y_{i}^{\prime R} & =\frac{\left[R_{i}-1.596\left(C r_{s}-128\right)\right]}{1.164}+16 \\
Y_{i}^{\prime G} & =\frac{\left[G_{i}+0.391\left(C b_{s}-128\right)+0.813\left(C r_{s}-128\right)\right]}{1.164}+16 \\
Y_{i}^{\prime B} & =\frac{\left[B_{i}-2.018\left(C b_{s}-128\right)\right]}{1.164}+16
\end{aligned}
$$

where $Y_{i}^{\prime R}, Y_{i}^{\prime G}$, and $Y_{i}^{\prime B}$ denote the exact luma modification solutions for the first, second, third equations, respectively. However, it is intractable to determine the unique luma modification solution of $Y_{i}^{\prime}$ that satisfies the three equations in Eq. (3) simultaneously. Therefore, in the luma modification (LM) method for CSFLM [4], the best luma modification solution of $Y_{i}^{\prime}$ is determined by examining all luma values in the search interval $\left[\operatorname{Low}_{i}, H_{i g h}\right]$ with $\operatorname{Low}_{i}=\left\lfloor\min \left(Y_{i}^{\prime R}, Y_{i}^{\prime R}, Y_{i}^{\prime R}\right)\right\rfloor$ and $H i g h_{i}=\left\lceil\max \left(Y_{i}^{\prime R}, Y_{i}^{\prime R}, Y_{i}^{\prime R}\right)\right\rceil$, where $\lceil$.$\rceil and \lfloor$.$\rfloor denote$ the ceiling and floor operations, respectively, such that the minimal COPY-based pixel-distortion value is achieved.

Based on the IMAX, Kodak, and SCI (screen content images) datasets, the experimental data indicated that the average number of the luma values examined in the search interval is 4.66 , and the execution time overhead required in the LM method is only $6.4 \%$ relative to that required to complete the 4:2:0(A) chroma subsampling and the encoding process in HEVC. Experimental results demonstrated the quality enhancement and quality-bitrate tradeoff merits of the LM method in the CSFLM scheme.

Although the search interval is short for the pixel in the smooth area, it may be long for the pixel in the textural area. Therefore, applying a differentiation technique on the search interval [4], Lin et al. [11] proposed a luma optimization (LO) method to reduce the number of luma values examined in the search interval. However, due to the differentiation technique used, the luma values examined in [11] are real values, so it still needs to take a floor operation and a ceiling operation on each examined luma value, and then a better luma modification solution is selected. With competitive quality performance, the LO method is faster than the LM method. Zhu et al. [21] deployed the LM idea [4] in their DCT (discrete cosine transform) based color cross-space distortion minimization-based image compression method to compensate for the residual between the original image and the reconstructed image, where the residual is caused by quantization and dequantization in JPEG. In [20], Zhu et al. proposed a cholesky decomposition approach to compress the color image by combining the chroma subsampling and luma modification.

As depicted in Fig. 1, after performing the luma modification for the whole luma image at the server side, the subsampled $\mathrm{CbCr}$ image and the modified luma image are fed into the encoder for compression. After transmitting the compressed subsampled chroma image and the luma image to the receiver via the communication network, at the client side, the decompressed subsampled $\mathrm{CbCr}$ image is upsampled, and then by Eq. (2), the upsampled $\mathrm{YCbCr}$ image is converted into a reconstructed RGB full-color image.

\section{CONTRIBUTIONS}

Relative to the current two methods [4], [11], the three contributions of the proposed nonlinear interpolation-based, namely the bicubic convolution interpolation (BCI)-based, 


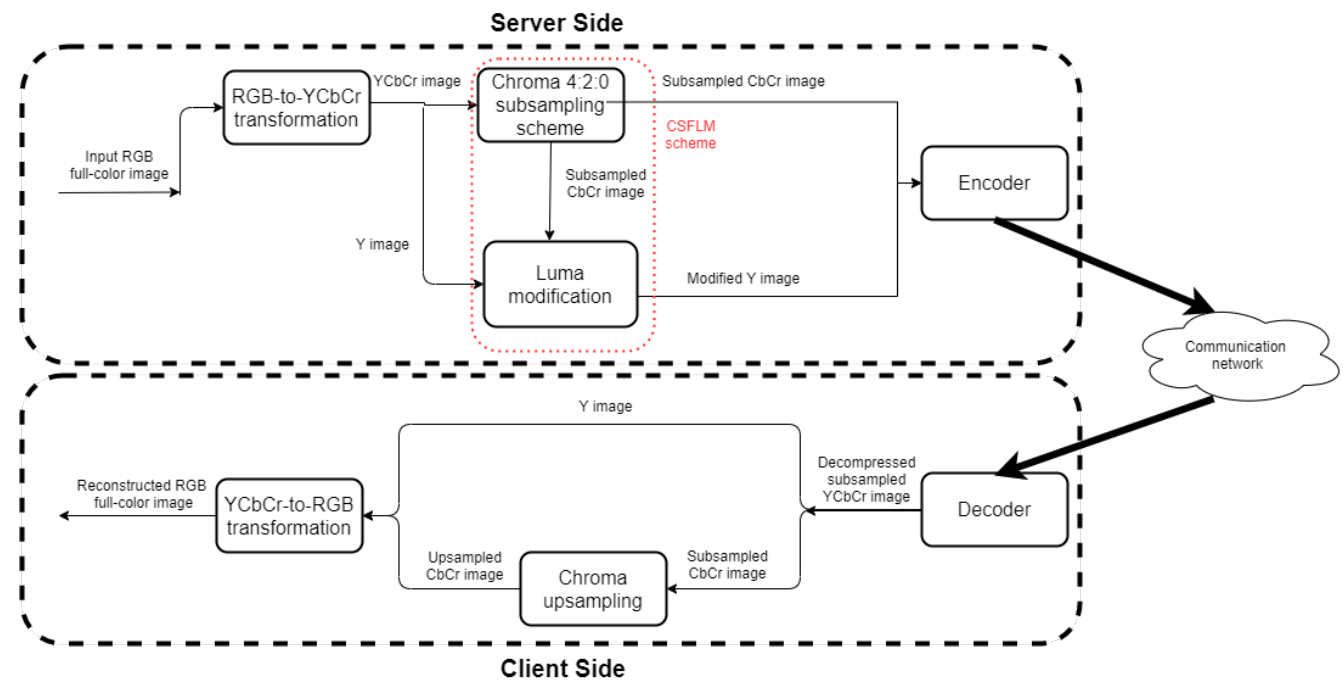

FIGURE 1. The CSFLM scheme in the coding system.

iterative luma modification method for $I^{R G B}$ are clarified as follows.

\section{1) The first contribution}

Differing from the COPY-based pixel-distortion function [4], [11], we propose a new and more effective BCI-based pixeldistortion function. Further, the convex property of the proposed distortion function, which serves as the base of the initial luma modification solution, is proved.

\section{2) The second contribution}

Differing from the search ways used in the LM and LO methods, in this paper, we model the luma modification problem for CSFLM as a BCI-based overdetermined system, and then we apply a pseudoinverse technique to obtain the initial luma modification solution. Furthermore, an iterative luma modification method is proposed to improve the initial luma modification solution, achieving substantial quality, execution time, and quality-bitrate tradeoff improvements.

\section{3) The third contribution}

Based on the Kodak [10], IMAX [7], SCI (screen content images) [14], CI (classical images) [20], [21], and Video [17] datasets, the thorough experimental results have demonstrated that on the newly released Versatile Video Coding (VVC) platform VTM-12.0 [18], our BCI-based iterative luma modification method achieves substantial quality, execution time, and quality-bitrate tradeoff improvements when compared with the LM method [4] and the LO method [11]. The considered quality metrics include the CPSNR (color peak signal-to-noise ratio), SSIM (structural similarity index measure) [19], and visual effect. The quality-bitrate tradeoff metric used is the BD-rate (Bjøntegaard delta bitrate) [1].

The rest of this paper is organized as follows. In Section III the proposed BCI-based pixel-distortion function is presented, and then we prove that the proposed pixel-distortion function is a convex function. In Section III, an iterative luma modification method is proposed. In Section [IV] the thorough experimental results are illustrated to justify the substantial quality, execution time, and quality-bitrate tradeoff improvements of our method. In Section $\square$ s some concluding remarks are made.

\section{THE PROPOSED NEW BCI-BASED PIXEL-DISTORTION FUNCTION}

In the CSFLM scheme of Fig. 1] the subsampled $(\mathrm{Cb}, \mathrm{Cr})$ pair of each $B^{C b C r}$ is known in advance and it is denoted by $\left(C b_{s}, C r_{s}\right)$. In this section, we first propose a novel and more effective BCI-based pixel-distortion function to measure the sum of squared errors (SSE) between the ground truth triple-value $\left(R_{i}, G_{i}, B_{i}\right)$ and the estimated analogue $\left(R_{i}^{\text {est }}, G_{i}^{\text {est }}, B_{i}^{\text {est }}\right), 1 \leq i \leq 4$. Next, we prove that the proposed pixel-distortion function is a convex function which serves as the base of the initial luma modification solution in our iterative method.

\section{A. THE PROPOSED PIXEL-DISTORTION FUNCTION}

Before estimating the triple-value $\left(R_{i}^{\text {est }}, G_{i}^{\text {est }}, B_{i}^{\text {est }}\right), 1 \leq$ $i \leq 4$, we first estimate each entry of $B^{e s t, C b C r}$, namely $\left(C b_{i}^{\text {est }}, C r_{i}^{\text {est }}\right)$.

\section{1) The estimation of $\left(\mathrm{Cb}_{i}^{\text {est }}, C r_{i}^{\text {est }}\right)$}

Without the loss of generality, we only describe how to apply our BCI-based estimation method to estimate $C b_{1}^{e s t}$ using the subsampled $\mathrm{Cb}$ value of $B^{C b}$, namely $C b_{s}$, and the subsampled $\mathrm{Cb}$ values of the neighboring $2 \times 2 \mathrm{Cb}$ blocks.

As depicted in Fig. 22(a), each $2 \times 2$ Cb block $B^{C b}$ is viewed as a $1 \times 1$ macro pixel marked in blue. As the origin of the $x y-$ coordinate system, the location of the subsampled $\mathrm{Cb}$ value of $B^{C b}$, namely $C b_{s}$, is set to $(0,0)$. Therefore, $C b_{1}^{\text {est }}$ marked in red is located at $(-0.25,0.25)$. 
Let $d_{i, j}^{h}$ and $d_{i, j}^{v}$ denote the horizontal and vertical distances between the reference subsampled $\mathrm{Cb}$ point, namely $\mathrm{Cb}(i, j)$, and the point $C b_{1}^{e s t}$ in the x-coordinate and ycoordinate, respectively. As depicted in Fig. 2 (b), for $\mathrm{Cb}(-1$, $1)$, the values of $d_{-1,1}^{h}$ and $d_{-1,1}^{v}$ are $-0.75(=-1+0.25)$ and $0.75(=1-0.25)$, respectively. Similarly, for $\mathrm{Cb}(0,0)\left(=C b_{s}\right)$, it yields $d_{0,0}^{h}=0.25(=0+0.25)$ and $d_{0,0}^{v}=-0.25(=0-0.25)$.

After defining $d_{i, j}^{h}$ and $d_{i, j}^{v}$, we deploy the bicubic convolution interpolation [9] in our BCI-based estimation method for estimating $C b_{1}^{e s t}$. The weight assigned to each reference subsampled $\mathrm{Cb}$ point, namely $C b(i, j)$, is given by

$$
\bar{W}(i, j)=W\left(d_{i, j}^{h}\right) W\left(d_{i, j}^{v}\right)
$$

with

$W(d)= \begin{cases}(p+2)|d|^{3}-(p+3)|d|^{2}+1 & \text { for }|d| \leq 1 \\ p|d|^{3}-5 p|d|^{2}+8 p|d|-4 p & \text { for } 1<|d|<2 \\ 0 & \text { otherwise. }\end{cases}$

where in our experiment, the best choice of the parameter $p$ in Eq. (5) is -0.5 . Due to the constraint: $|d|<2$, for estimating $C b_{1}^{\text {est }}$, as depicted in Fig. 2(a), only sixteen reference subsampled $\mathrm{Cb}$ values are considered.

Therefore, $C b_{1}^{\text {est }}$ can be estimated by

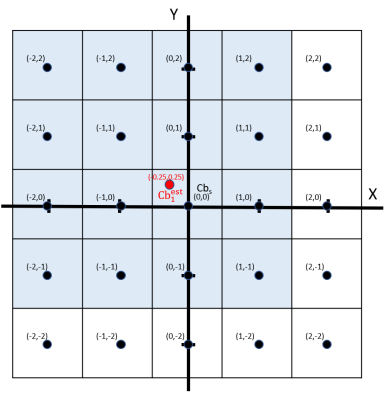

(a)

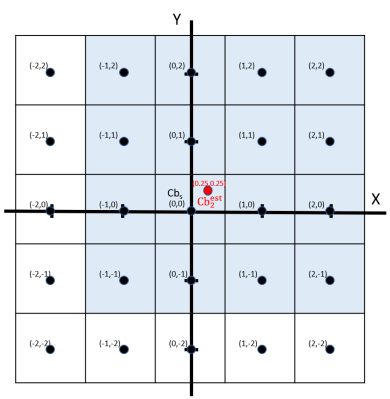

(c)

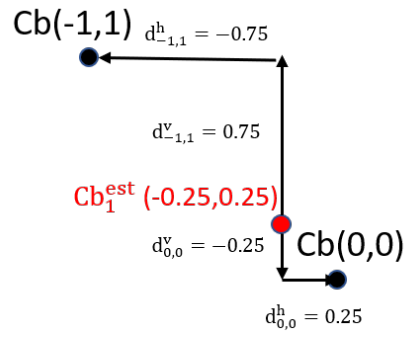

(b)

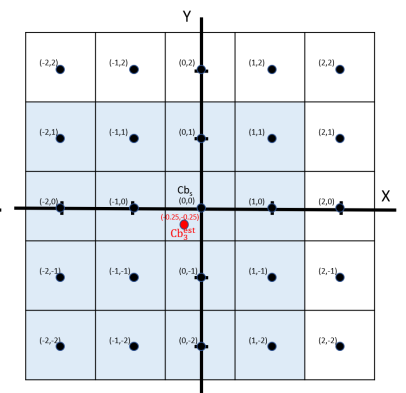

(d)

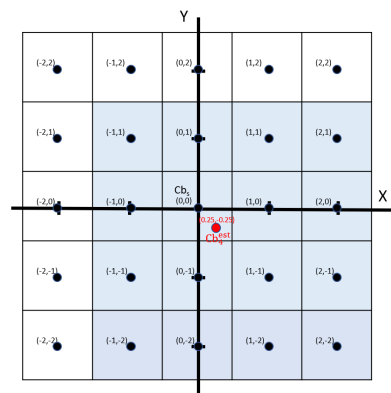

(e)

where $\bar{W}(0.25)=\bar{W}(-0.25)=0.8672$. All values of $\bar{W}(i, j)$ for $-2 \leq \mathrm{i} \leq 1$ and $-1 \leq \mathrm{j} \leq 2$ have been calculated in advance and they have been stored in a lookup table.

\section{2) The proposed pixel-distortion function}

Before presenting our new pixel-distortion function, we first estimate the estimated $i$ th RGB triple-value $\left(R_{i}^{\text {est }}, G_{i}^{\text {est }}\right.$, $\left.B_{i}^{\text {est }}\right), 1 \leq i \leq 4$. Based on the parameter $Y_{i}^{\prime}$ and the two estimated chroma values, namely $C b_{i}^{e s t}$ and $C r_{i}^{e s t}$, by Eq. (2), the estimated $i$ th RGB triple-value, $\left(R_{i}^{\text {est }}, G_{i}^{\text {est }}\right.$, $B_{i}^{\text {est }}$ ), can be obtained by the following YCbCr-to-RGB color conversion:

$$
\left[\begin{array}{l}
R_{i}^{\text {est }} \\
G_{i}^{\text {est }} \\
B_{i}^{\text {est }}
\end{array}\right]=\left[\begin{array}{ccc}
1.164 & 0 & 1.596 \\
1.164 & -0.391 & -0.813 \\
1.164 & 2.018 & 0
\end{array}\right]\left[\begin{array}{c}
Y_{i}^{\prime}-16 \\
C b_{i}^{\text {est }}-128 \\
C r_{i}^{\text {est }}-128
\end{array}\right]
$$

Using the SSE metric, the proposed RGB full-color pixeldistortion between the ground truth pixel $\left(R_{i}, G_{i}, B_{i}\right), 1 \leq$ $i \leq 4$, and the estimated pixel $\left(R_{i}^{e s t}, G_{i}^{\text {est }}, B_{i}^{\text {est }}\right)$ is expressed as
FIGURE 2. The sixteen reference subsampled $\mathrm{Cb}$ values for estimating $C b_{i}^{\text {est }}$ for $1 \leq i \leq 4$. (a) The sixteen reference subsampled $\mathrm{Cb}$ values in blue for $C b_{1}^{e s t}$. (b) The values of $d_{-1,1}^{h}, d_{-1,1}^{v}, d_{0,0}^{h}$, and $d_{0,0}^{v}$. (c) The 16 reference subsampled $\mathrm{Cb}$ values in blue for estimating $C b_{2}^{\text {est }}$. (d) The 16 reference subsampled $\mathrm{Cb}$ values in blue for estimating $C b_{3}^{\text {est }}$. (e) The 16 reference subsampled $\mathrm{Cb}$ values in blue for estimating $\mathrm{Cb}_{4}^{\text {est }}$.

$$
\begin{aligned}
P D\left(Y_{i}^{\prime}\right)= & {\left[\left(R_{i}-R_{i}^{e s t}\right)^{2}+\left(G_{i}-G_{i}^{e s t}\right)^{2}+\left(B_{i}-B_{i}^{e s t}\right)^{2}\right] } \\
= & \left\{R_{i}-\left[1.164\left(Y_{i}^{\prime}-16\right)+1.596\left(C r_{i}^{e s t}-128\right)\right]\right\}^{2} \\
& +\left\{G_{i}-\left[1.164\left(Y_{i}^{\prime}-16\right)-0.391\left(C b_{i}^{e s t}-128\right)\right.\right. \\
& \left.\left.-0.813\left(C r_{i}^{e s t}-128\right)\right]\right\}^{2} \\
& +\left\{B_{i}-\left[1.164\left(Y_{i}^{\prime}-16\right)+2.018\left(C b_{i}^{\text {est }}-128\right)\right]\right\}^{2} \\
= & \left\{\text { Const }_{1}-\left[1.164\left(Y_{i}^{\prime}\right)\right]\right\}^{2} \\
& +\left\{\text { Const }_{2}-\left[1.164\left(Y_{i}^{\prime}\right)\right]\right\}^{2} \\
& +\left\{\text { Const }_{3}-\left[1.164\left(Y_{i}^{\prime}\right)\right]\right\}^{2}
\end{aligned}
$$

where Const $_{1}=R_{i}+1.164 * 16+1.596\left(\right.$ Cr $\left._{i}^{\text {est }}-128\right)$, Const $_{2}=G_{i}+1.164 * 16-0.391\left(C b_{i}^{\text {est }}-128\right)-$ $0.813\left(C_{i}^{\text {est }}-128\right)$, and Const $_{3}=B_{i}+1.164 * 16+$ $2.018\left(C b_{i}^{\text {est }}-128\right)$. From Eq. 88, we know that the proposed 
pixel-distortion function $P D\left(Y_{i}^{\prime}\right)$ is a quadratic function with the parameter $Y_{i}^{\prime}$.

In what follows, we prove that $P D\left(Y_{i}^{\prime}\right)$ is a convex function, and this convex property serves as the base of the initial luma modification solution in our iterative luma modification method.

\section{B. CONVEX PROPERTY PROOF OF THE PROPOSED PIXEL-DISTORTION FUNCTION}

According to the convex function definition [2], if a function $f$ satisfies $f(\theta x+(1-\theta) y) \leq \theta f(x)+(1-\theta) f(y)$ for $0 \leq \theta \leq 1$, where the two points, $x$ and $y$, are taken from the function $f$, then $f$ is called a convex function.

We now prove that our new pixel-distortion function $P D\left(Y_{i}^{\prime}\right), 1 \leq i \leq 4$, in Eq. (8) is a convex function because it satisfies the condition: " $P D\left(\theta Y_{i_{1}}^{\prime}+(1-\theta) Y_{i_{2}}^{\prime}\right) \leq$ $\theta P D\left(Y_{i_{1}}^{\prime}\right)+(1-\theta) P D\left(Y_{i_{2}}^{\prime}\right)$," where any two points, namely $Y_{i_{1}}^{\prime}$ and $Y_{i_{2}}^{\prime}$, are taken from $P D\left(Y_{i}^{\prime}\right)$. By Eq. (8), the left hand side in the inequality of the above-mentioned condition is expressed as

$$
\begin{aligned}
P D\left(\theta Y_{i_{1}}^{\prime}+(1-\theta) Y_{i_{2}}^{\prime}\right)= & \left\{\text { Const }_{1}-\left[1.164\left(\theta Y_{i_{1}}^{\prime}+(1-\theta) Y_{i_{2}}^{\prime}\right)\right]\right\}^{2} \\
& +\left\{\text { Const }_{2}-\left[1.164\left(\theta Y_{i_{1}}^{\prime}+(1-\theta) Y_{i_{2}}^{\prime}\right)\right]\right\}^{2} \\
& +\left\{\text { Const }_{3}-\left[1.164\left(\theta Y_{i_{1}}^{\prime}+(1-\theta) Y_{i_{2}}^{\prime}\right)\right]\right\}^{2}
\end{aligned}
$$

where the three constant terms, namely Const $_{1}$, Const $_{2}$, and Const $_{3}$, have been defined in Eq. 87. Expanding the right hand side of Eq. (9), it yields

$$
\begin{array}{rl}
P & D\left(\theta Y_{i_{1}}^{\prime}+(1-\theta) Y_{i_{2}}^{\prime}\right) \\
= & \left\{\text { Const }_{1}^{2}-2.328\left(\text { Const }_{1}\right)\left(\theta Y_{i_{1}}^{\prime}+(1-\theta) Y_{i_{2}}^{\prime}\right)\right. \\
& \left.+\left(1.164\left(\theta Y_{i_{1}}^{\prime}+(1-\theta) Y_{i_{2}}^{\prime}\right)\right)^{2}\right\} \\
& +\left\{\text { Const }_{2}^{2}-2.328\left(\text { Const }_{2}\right)\left(\theta Y_{i_{1}}^{\prime}+(1-\theta) Y_{i_{2}}^{\prime}\right)\right. \\
& \left.+\left(1.164\left(\theta Y_{i_{1}}^{\prime}+(1-\theta) Y_{i_{2}}^{\prime}\right)\right)^{2}\right\} \\
& +\left\{\text { Const }_{3}^{2}-2.328\left(\text { Const }_{3}\right)\left(\theta Y_{i_{1}}^{\prime}+(1-\theta) Y_{i_{2}}^{\prime}\right)\right. \\
& \left.+\left(1.164\left(\theta Y_{i_{1}}^{\prime}+(1-\theta) Y_{i_{2}}^{\prime}\right)\right)^{2}\right\}
\end{array}
$$

By the same argument, the right hand side in the inequality of the above-mentioned convex function condition is expressed as

$$
\begin{aligned}
\theta & P\left(Y_{i_{1}}^{\prime}\right)+(1-\theta) P D\left(Y_{i_{2}}^{\prime}\right) \\
= & \theta\left\{\text { Const }_{1}-\left[1.164\left(Y_{i_{1}}^{\prime}\right)\right]\right\}^{2} \\
& +(1-\theta)\left\{\text { Const }_{1}-\left[1.164\left(Y_{i_{2}}^{\prime}\right)\right]\right\}^{2} \\
& +\theta\left\{\text { Const }_{2}-\left[1.164\left(Y_{i_{1}}^{\prime}\right)\right]\right\}^{2} \\
& +(1-\theta)\left\{\text { Const }_{2}-\left[1.164\left(Y_{i_{2}}^{\prime}\right)\right]\right\}^{2} \\
& +\theta\left\{\text { Const }_{3}-\left[1.164\left(Y_{i_{1}}^{\prime}\right]\right\}^{2}\right. \\
& +(1-\theta)\left\{\text { Const }_{3}-\left[1.164\left(Y_{i_{2}}^{\prime}\right)\right]\right\}^{2} \\
= & \left\{\text { Const }_{1}^{2}-2.328 \text { Const }_{1}\left(\theta Y_{i_{1}}^{\prime}+(1-\theta) Y_{i_{2}}^{\prime}\right)\right. \\
& \left.+\theta\left(1.164 Y_{i_{1}}^{\prime}\right)^{2}+(1-\theta)\left(1.164 Y_{i_{2}}^{\prime}\right)^{2}\right\} \\
& +\left\{\text { Const }_{2}^{2}-2.328 \text { Const }_{2}\left(\theta Y_{i_{1}}^{\prime}+(1-\theta) Y_{i_{2}}^{\prime}\right)\right. \\
& \left.+\theta\left(1.164 Y_{i_{1}}^{\prime}\right)^{2}+(1-\theta)\left(1.164 Y_{i_{2}}^{\prime}\right)^{2}\right\} \\
& +\left\{\text { Const }_{3}^{2}-2.328 \text { Const }_{3}\left(\theta Y_{i_{1}}^{\prime}+(1-\theta) Y_{i_{2}}^{\prime}\right)\right. \\
& \left.+\theta\left(1.164 Y_{i_{1}}^{\prime}\right)^{2}+(1-\theta)\left(1.164 Y_{i_{2}}^{\prime}\right)^{2}\right\}
\end{aligned}
$$

Subtracting Eq. (10) from Eq. (11) yields

$$
\begin{aligned}
& \theta P D\left(Y_{i_{1}}^{\prime}\right)+(1-\theta) P D\left(Y_{i_{2}}^{\prime}\right)-P D\left(\theta Y_{i_{1}}^{\prime}+(1-\theta) Y_{i_{2}}^{\prime}\right) \\
& =\left\{\theta\left(1.164 Y_{i_{1}}^{\prime}\right)^{2}+(1-\theta)\left(1.164 Y_{i_{2}}^{\prime}\right)^{2}-\left(1.164\left(\theta Y_{i_{1}}^{\prime}+(1-\theta) Y_{i_{2}}^{\prime}\right)\right)^{2}\right\} \\
& +\left\{\theta\left(1.164 Y_{i_{1}}^{\prime}\right)^{2}+(1-\theta)\left(1.164 Y_{i_{2}}^{\prime}\right)^{2}-\left(1.164\left(\theta Y_{i_{1}}^{\prime}+(1-\theta) Y_{i_{2}}^{\prime}\right)\right)^{2}\right\} \\
& +\left\{\theta\left(1.164 Y_{i_{1}}^{\prime}\right)^{2}+(1-\theta)\left(1.164 Y_{i_{2}}^{\prime}\right)^{2}-\left(1.164\left(\theta Y_{i_{1}}^{\prime}+(1-\theta) Y_{i_{2}}^{\prime}\right)\right)^{2}\right\} \\
& =3(1.164)^{2}\left\{\left(\theta-\theta^{2}\right) Y_{i_{1}}^{\prime}{ }^{2}+\left(\theta-\theta^{2}\right) Y_{i_{2}}^{\prime}{ }^{2}-2\left(\theta-\theta^{2}\right) Y_{i_{1}}^{\prime} Y_{i_{2}}^{\prime}\right\} \\
& =3(1.164)^{2}\left(\theta-\theta^{2}\right)\left\{Y_{i_{1}}^{\prime}-Y_{i_{2}}^{\prime}\right\}^{2} \geq 0
\end{aligned}
$$

Eq. (12) implies that the convex function condition " $P D\left(\theta Y_{i_{1}}^{\prime}+(1-\theta) Y_{i_{2}}^{\prime}\right) \leq \theta P D\left(Y_{i_{1}}^{\prime}\right)+(1-\theta) P D\left(Y_{i_{2}}^{\prime}\right) "$ holds to our new proposed pixel-distortion function $P D\left(Y_{i}^{\prime}\right)$ in Eq. (8). We thus have the following result.

Proposition 1. Our new pixel-distortion function $P D\left(Y_{i}^{\prime}\right)$ in Eq. (8) is a convex function.

\section{THE PROPOSED FAST AND EFFECTIVE ITERATIVE LUMA MODIFICATION METHOD}

We first transform the luma modification problem in CSFLM to an overdetermined system. Next, we apply the pseudoinverse technique to determine the initial integer luma modification solution. By Proposition 1, we propose a fast and effective iterative luma modification method to improve the initial integer luma modification solution.

\section{A. DETERMINING THE INITIAL INTEGER LUMA MODIFICATION SOLUTION}

Ideally, we hope to determine the luma modification solution of $Y_{i}^{\prime}$ such that the resultant triple-value $\left(R_{i}^{\text {est }}, G_{i}^{\text {est }}, B_{i}^{\text {est }}\right)$ in the left side of Eq. (7) could be equal to the original RGB triple-value $\left(R_{i}, G_{i}, B_{i}\right)$. Accordingly, we consider the three equations: 


$$
\begin{aligned}
R_{i} & =R_{i}^{e s t} \\
& =1.164\left(Y_{i}^{\prime}-16\right)+1.596\left(C r_{i}^{e s t}-128\right) \\
G_{i} & =G_{i}^{e s t} \\
& =1.164\left(Y_{i}^{\prime}-16\right)-0.391\left(C b_{i}^{e s t}-128\right)-0.813\left(C r_{i}^{e s t}-128\right) \\
B_{i} & =B_{i}^{\text {est }} \\
& =1.164\left(Y_{i}^{\prime}-16\right)+2.018\left(C b_{i}^{\text {est }}-128\right)
\end{aligned}
$$

In the overdetermined system of Eq. (13), there are three equations, but there is only one parameter $Y_{i}^{\prime}$. To solve $Y_{i}^{\prime}$ in Eq. (13), we first move the constant terms in the right hand side of each equality in Eq. (13) to the left hand side. Let the three constant terms be denoted by a $3 \times 1$ vector, namely $\left(\bar{R}_{i}, \bar{G}_{i}, \bar{B}_{i}\right)^{t}=\left(R_{i}-1.596\left(C r_{i}^{e s t}\right)+222.912, G_{i}+\right.$ $0.391\left(C b_{i}^{\text {est }}\right)+0.831\left(C b_{i}^{\text {est }}\right)-135.488, B_{i}-2.018\left(C b_{i}^{\text {est }}\right)+$ $276.928)^{t}$. Eq. 13 is thus expressed as

$$
\begin{aligned}
{\left[\begin{array}{c}
\overline{\bar{R}}_{i} \\
\overline{\bar{G}}_{i} \\
\bar{B}_{i}
\end{array}\right]=\left[\begin{array}{c}
R_{i}-1.596\left(C r_{i}^{\text {est }}\right)+222.912 \\
G_{i}+0.391\left(C b_{i}^{\text {est }}\right)+0.831\left(C b_{i}^{\text {est }}\right)-135.488 \\
B_{i}-2.018\left(C b_{i}^{\text {est }}\right)+276.928
\end{array}\right] } \\
=\left[\begin{array}{c}
1.164 \\
1.164 \\
1.164
\end{array}\right]\left[Y_{i}^{\prime}\right]
\end{aligned}
$$

Let $b=\left(\bar{R}_{i}, \bar{G}_{i}, \bar{B}_{i}\right)^{t}$ and $A=(1.164,1.164,1.164)^{t}$. Eq. (14) is expressed as

$$
A Y_{i}^{\prime}=b
$$

We apply the pseudo-inverse technique to solve $Y_{i}^{\prime}$ in Eq. 15. Multiplying both sides of Eq. (15) by $A^{t}$, it yields $A^{t} A Y_{i}^{\prime}=A^{t} b$, and then multiplying $\left(A^{t} A\right)^{-1}$ to both sides of $A^{t} A Y_{i}^{\prime}=A^{t} b$, it yields

$$
Y_{i}^{\prime}=\left(A^{t} A\right)^{-1} A^{t} b
$$

Putting $\left(A^{t} A\right)^{-1}=\frac{1}{3 *(1.164)^{2}}$ and $A^{t}=(1.164,1.164,1.164)$ back to Eq. (16), the initial real luma modification solution for $Y_{i}^{\prime}$ is given by

$$
\begin{aligned}
Y_{i}^{\prime} & =\frac{1}{3 *(1.164)^{2}}(1.164,1.164,1.164) b \\
& =(0.286,0.286,0.286) b \\
& =0.286\left(R_{i}+G_{i}+B_{i}\right)-0.224 C r_{i}^{\text {est }}-0.466 C b_{i}^{\text {est }} \\
& +104.34
\end{aligned}
$$

where the values of $R_{i}, G_{i}$, and $B_{i}$ are known; the values of $C b_{i}^{e s t}$ and $C r_{i}^{\text {est }}$ have been calculated using Eq. (6) or its analogue. Considering the practical integer domain, we take the ceiling and flooring operations on the initial real luma modification solution in Eq. (17) to obtain two possible integer luma modification solutions, namely $L=$ $\lfloor(0.286,0.286,0.286) b\rfloor$ and $H=\lceil(0.286,0.286,0.286) b\rceil$, where $H=L+1$. Next, we select the best one, $L$ or $H$, with the minimal pixel-distortion value as the initial integer luma modification solution, namely $Y_{i}^{\prime(0)}$.

\section{B. THE PROPOSED ITERATIVE LUMA MODIFICATION METHOD}

For easy readability, we take a practical example to sketch the room to improve the initial integer luma modification solution using our iterative method. Given a practical example in Fig. 3 a), the 16 reference subsampled chroma pairs are shown in the $4 \times 4$ macro block, where the subsampled $(\mathrm{Cb}, \mathrm{Cr})$-pair of the current $\mathrm{CbCr}$ block $\mathrm{B}^{\mathrm{CbCr}}$ is denoted by $\left(C b_{s}, C r_{s}\right)(=(109,136))$, and the considered luma modification parameter $Y_{2}^{\prime}$ is marked by a black bullet.

Using an analogue of Eq. (6) to estimate $C b_{2}^{e s t}$ and $C r_{2}^{e s t}$, it yields $C b_{2}^{e s t}=116$ and $C r_{2}^{e s t}=134$. It is known that $R_{2}=26, G_{2}=15$, and $B_{2}=2$. Furthermore, by Eq. (8), the pixel-distortion function $P D\left(Y_{2}^{\prime}\right)$ is expressed as

$$
\begin{aligned}
P D\left(Y_{2}^{\prime}\right) & =\left(R_{2}-\left[1.164 Y_{2}^{\prime}+1.596 C r_{2}^{\text {est }}-222.912\right]\right)^{2} \\
& +\left(G_{2}-\left[1.164 Y_{2}^{\prime}-0.391 C b_{2}^{\text {est }}-0.813 C r_{2}^{\text {est }}\right.\right. \\
& +135.488])^{2}+\left(B_{2}-\left[1.164 Y_{2}^{\prime}+2.018 C b_{2}^{\text {est }}\right.\right. \\
& -276.928])^{2} \\
& =\left(35.048-1.164 Y_{2}^{\prime}\right)^{2}+\left(33.81-1.164 Y_{2}^{\prime}\right)^{2} \\
& +\left(44.84-1.164 Y_{2}^{\prime}\right)^{2}
\end{aligned}
$$

In the real domain, the plot of the convex function $P D\left(Y_{2}^{\prime}\right)$ in Eq. (18) is depicted in Fig. 33 b).

Considering the interval $[25,39]$ for $Y_{2}^{\prime}$ in Fig. 3 b), we cut off the corresponding convex curve segment, and then the enlarged discrete plot of the curve segment is depicted in Fig. 3 (c). In Fig. 3. c), the path from the point $Y_{2}^{\prime(0)}$ to $Y_{2}^{\prime(2)}$ indicates the room to improve the initial integer luma modification solution, where the iteration number used in our iterative method is 2 .

To refine the initial integer luma modification solution $Y_{2}^{\prime(0)}$, for easy exposition, we assume $Y_{2}^{\prime(0)}=\left\lfloor Y_{2}^{\prime(0)}\right\rfloor=L$. In the example of Fig. 3. (c), the initial integer luma modification solution $P D\left(Y_{2}^{\prime}\right)$ equals 32 and the pixel-distortion value $P D(32)$ equals 17 which is marked in orange in Fig. 4 (a). Due to the assumption: $Y_{2}^{\prime(0)}=\left\lfloor Y_{2}^{\prime(0)}\right\rfloor=L$, we further consider the left neighboring luma modification solution 31 $\left(=Y_{2}^{\prime(0)}-1=32-1\right)$ as the refined luma modification solution candidate. Because the value of $\mathrm{PD}(31)$ equals 9, as marked in green in Fig. 4(a), the luma modification solution is refined from $Y_{2}^{\prime(0)}(=32)$ to $Y_{2}^{\prime(1)}(=31)$, and the pixeldistortion reduction is $8(=17-9)$.

By the same argument, in the second iteration, i.e. $k=2$, as depicted in Fig. 4(b), the luma modification solution is refined from 31 to 30 and the pixel-distortion reduction is $4(=9-5)$. The above iterative luma modification solutionrefinement process continues to the left until no improvement is achievable. It is notable that if $Y_{2}^{\prime(0)}=\left\lceil Y_{2}^{\prime(0)}\right\rceil=H$, the above iterative luma modification solution-refinement process continues to the right until no improvement is achievable.

For $Y_{2}^{\prime(0)}=\left\lfloor Y_{2}^{\prime(0)}\right\rfloor=L$, the proposed BCI-based iterative luma modification method, namely Algorithm 1, is listed 
below.

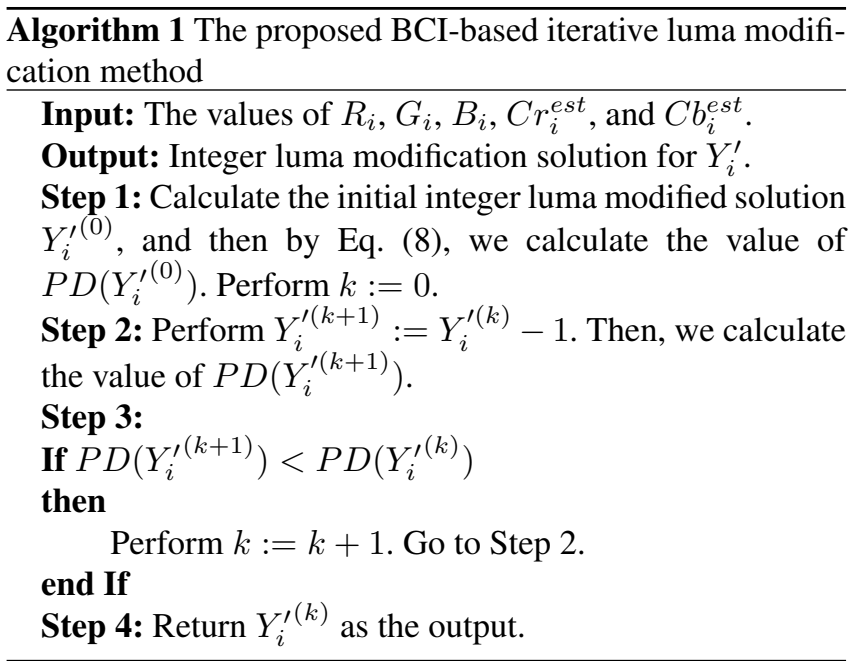

\section{EXPERIMENTAL RESULTS}

Based on the Kodak dataset with 24 images, the IMAX dataset with 18 images, the Video dataset with 200 images, the CI dataset with 8 images, and the SCI dataset with 20 images, the quality, execution time, and quality-bitrate tradeoff improvements of our iterative luma modification method are demonstrated when compared with the two state-of-theart methods [4], [11]. For convenience, the five considered chroma subsampling methods are denoted by the set $C_{s}=$ $\{4: 2: 0(\mathrm{~A}), 4: 2: 0(\mathrm{~L}), 4: 2: 0(\mathrm{R}), 4: 2: 0(\mathrm{D})$, Anchor $\}$; the three considered chroma upsampling methods are denoted by the set $C_{u}=\{\mathrm{NN}, 4$-tap, INTER_CUBIC $\}$ where the nearest neighbor (NN) method and the 4-tap method are supported by the VVC platform [18]. The INTER_CUBIC method is supported by the OpenCV library [3], [13].

All the concerned experiments are implemented on a computer with an Intel Core i7-9700 CPU 3.0 GHz and $32 \mathrm{~GB}$ RAM. The operating system is the Microsoft Windows 10 64- bit operating system. The program development environment is Visual C++ 2017. The VVC reference software platform used for compression is VTM-12.0. The execution code of our iterative luma modification method can be accessed from the website [5].

\section{A. QUALITY AND EXECUTION TIME IMPROVEMENTS OF OUR LUMA MODIFICATION METHOD}

When setting QP (quantization parameter) to zero, we take the two quality metrics, namely CPSNR and SSIM, to demonstrate the quality enhancement merit of our iterative luma modification method, abbreviated as 'Ours'.

CPSNR is used to evaluate the average quality of the reconstructed RGB full-color images for one dataset with $N$ images, and it is defined by

$$
\mathrm{CPSNR}=\frac{1}{N} \sum_{n=1}^{N} 10 \log _{10} \frac{255^{2}}{C M S E}
$$

with $C M S E=\frac{1}{3 W H} \sum_{p \in P} \sum_{c \in\{R, G, B\}}\left[I_{n, c}^{R G B}(p)-\right.$ $\left.I_{n, c}^{R G B}(p)\right]^{2}$ in which $\mathrm{P}=\{(x, y) \mid 1 \leq x \leq H, 1 \leq y \leq W\}$ denotes the set of pixel coordinates in one $W \times H$ image. $I_{n, c}^{R G B}(p)$ and $I_{n, c}^{R G B}(p)$ denote the c-color value of the pixel at position $p$ in the $n$th original RGB full-color image and the reconstructed analogue, respectively. The CPSNR value equals the mean of the five CPSNR values for the five datasets.

SSIM [19] is used to measure the joint preservation effects of luminance, contrast, and structure similarity between the original image and the reconstructed one. For $I^{R G B}$, the SSIM value is measured by the mean of the three SSIM values for the R, G, and B color planes.

\section{1) Quality enhancement merit}

To demonstrate the quality merit of our luma modification method "Ours", in Table 1, we first demonstrate the CPSNR and SSIM performance of all 15 combinations in $C_{s} \times L M \times$ $C_{u}$, where "LM" denotes the LM method in [4]. In the three combinations, namely $c_{s} \times L M \times C_{u}$, the combination " $c_{s}$-LM-INTER $R_{C U B I C}$ " always has the best CPSNR and SSIM performance, as shown in boldface. We thus select the five combinations " $c_{s}$-LM-INTER $R_{C U B I C}$ " as the comparative combinations. Similarly, for the 15 combinations in $C_{s} \times L O \times C_{u}$, where "LO" denotes the LO method in [11], we select five combinations " $C_{s} \times \mathrm{LO}-I N T E R_{C U B I C}$ " as the comparative combinations.

For comparison fairness, considering each $c_{s}$ in $C_{s}$, we compare our combination " $c_{s}$-Ours-INTER $R_{C U B I C}$ " with the two comparative combinations, namely $c_{s}$-LM$I N T E R_{C U B I C}$ and $c_{s}$-LO-INTE $R_{C U B I C}$, to justify the quality superiority of our luma modification method "Ours" over the LM method [4] and the LO method [11]. Based on the five datasets, for $\mathrm{QP}=0$, Table 2 indicates that the CPSNR gains of our five combinations over the corresponding five comparative combinations, namely $C_{s} \times$-LMINTER $R_{C U B I C}$, are $0.4644 \mathrm{~dB}, 0.8239 \mathrm{~dB}, 0.9274 \mathrm{~dB}$, $1.1261 \mathrm{~dB}$, and $0.6441 \mathrm{~dB}$, respectively. On average, the CPSNR gain of our luma modification method "Ours" over the LM method [4] is $0.7972 \mathrm{~dB}$. In the same table, we observe that the average CPSNR gain of our luma modification method over the LO method [11] is $0.7502 \mathrm{~dB}$.

Table 2 also indicates that the average SSIM gain of our luma modification method over the LM method and the LO method are 0.0023 and 0.0025 , respectively.

\section{2) Execution time merit}

Based on the five datasets, for one image, the average execution time required in the LM method [4] is 0.2049 seconds. The average execution time required in the LO method [11] is 0.1931 seconds. The average execution time required in our luma modification method is 0.1564 seconds. It indicates the execution time improvement merit of our method over the LM and LO methods. 


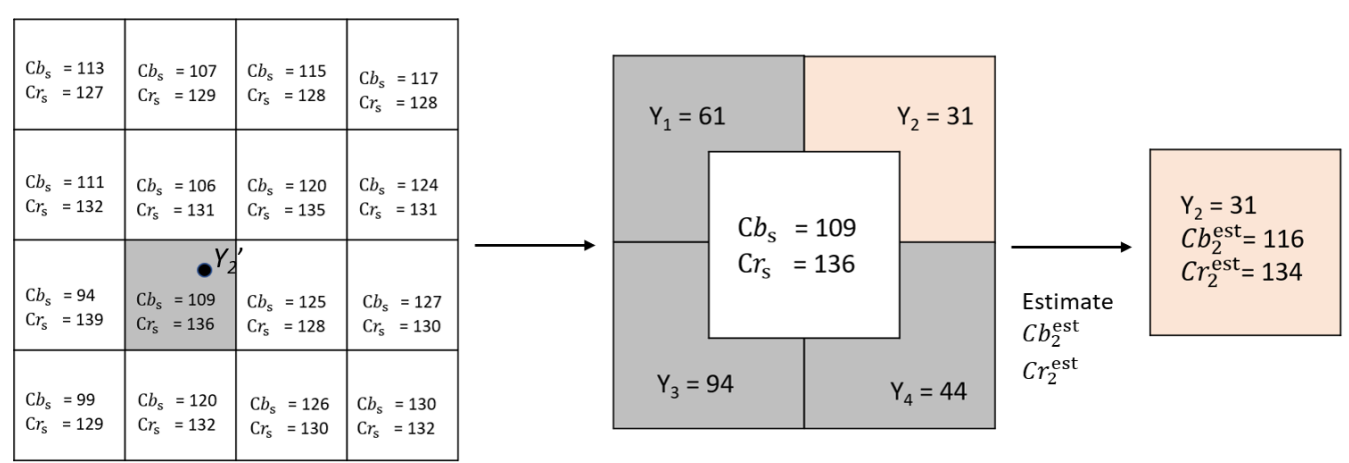

(a)

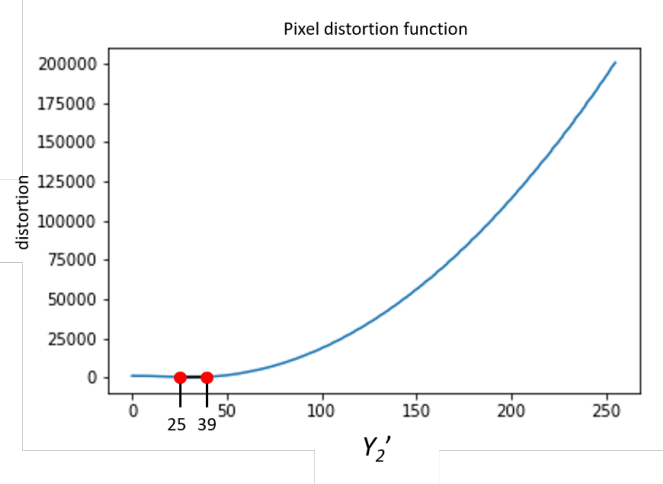

(b)

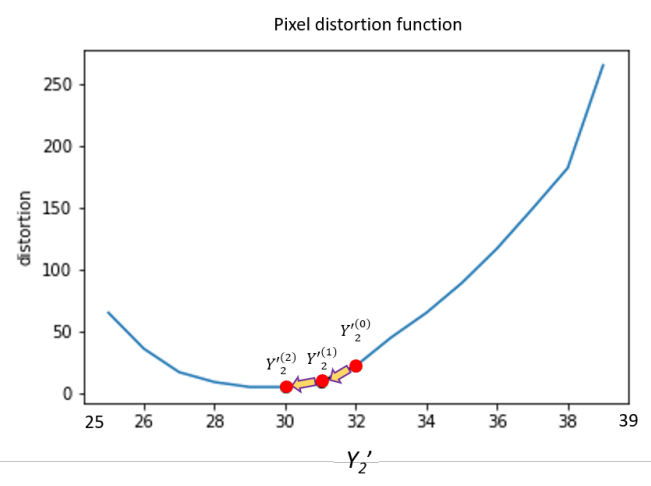

(c)

FIGURE 3. The sketch of the proposed iterative luma modification method. (a) An example for determining the luma modification solution for $Y_{2}^{\prime}$. (b) The plot of the convex pixel-distortion function $P D\left(Y_{2}^{\prime}\right)$ for Fig. 3. a) in the real domain. (c) The initial integer luma modification solution $Y_{2}^{\prime(0)}$ the refined solution $Y_{2}^{\prime(1)}$, and the refined solution $Y_{2}^{\prime(2)}$.

TABLE 1. THE CPSNR AND SSIM PERFORMANCE OF ALL COMBINATIONS IN $C_{s} \times \operatorname{LM}[4] \times C_{u}$ AND $C_{s} \times \operatorname{LO}[11] \times C_{u}$.

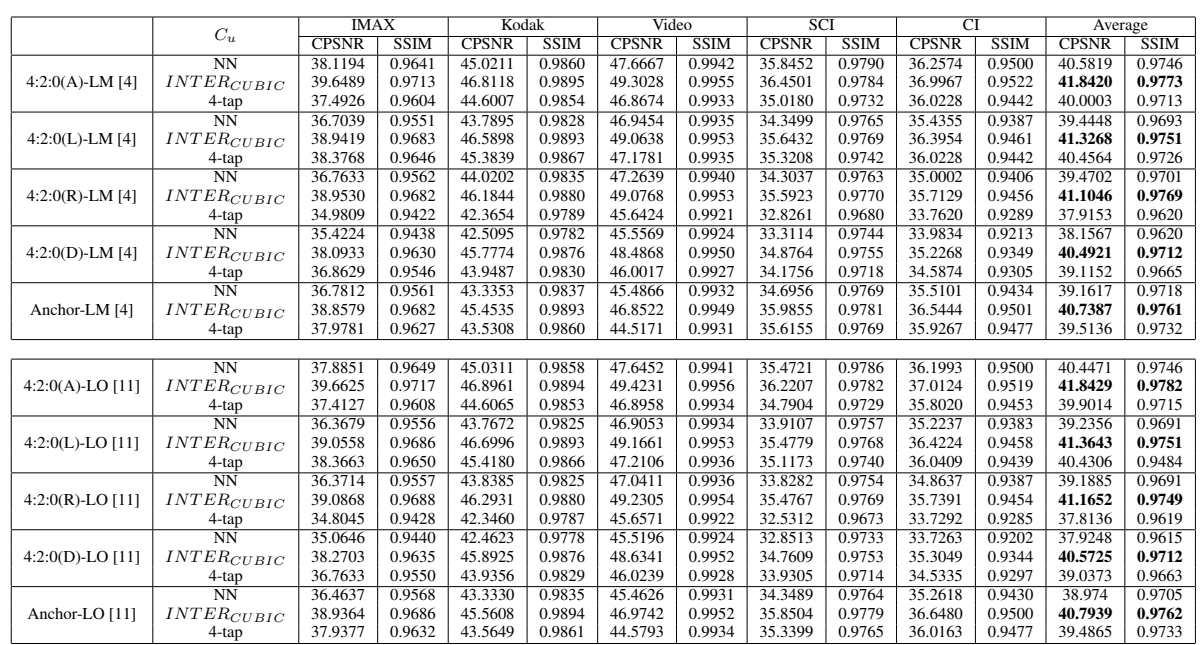




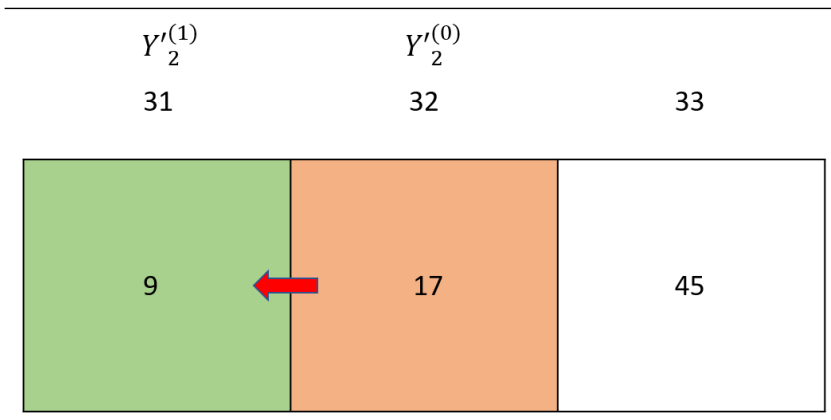

(a)

$\begin{array}{ccc}Y^{\prime(2)} & Y_{2}^{\prime(1)} & Y_{2}^{\prime(0)} \\ 30 & 31 & 32\end{array}$

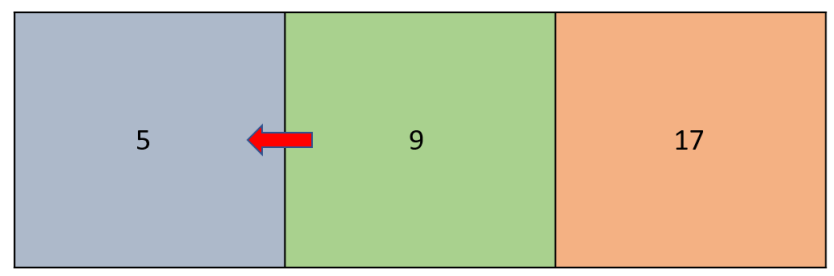

(b)

FIGURE 4. Two luma modification solution-refinement iterations. (a) First solution-refinement iteration. (b) Second solution-refinement iteration.

\section{B. VISUAL EFFECT AND BD-RATE MERITS OF OUR LUMA MODIFICATION METHOD}

In this subsection, the visual effect and the quality-bitrate tradeoff merist of our luma modification method are illustrated.

\section{1) The visual effect merit}

We first take the 5th ground truth SCI image in Fig. 5(a) to demonstrate the visual effect merit of the reconstructed RGB full-color image using our three combinations, namely 4:2:0(A)-Ours $\times C_{u}$, relative to the six comparative combinations, namely 4:2:0(A)-LM $\times C_{u}[4]$ and 4:2:0(A)-LO $\times C_{u}$ [11].

The magnified subimage in Fig. 5(b) is decoupled from the color region containing words "VIEW SCHEDULE" in Fig. 5 (a). For QP $=48$, after performing the 9 considered combinations on Fig. 5(b), the 9 reconstructed magnified subimages for Fig. 5(b) are shown in Figs. 5(c)-(k). From Figs. 5(c)-(k), we observe that our combination "4:2:0(A)ours- $c_{u}$ ", $c_{u} \in C_{u}$, always has a better visual effect than "4:2:0(A)-LM- $c_{u}$ " [4] and "4:2:0(A)-LO- $c_{u}$ " [11].

Next, we take the 18th ground truth IMAX image in Fig. 6(a) to demonstrate the visual effect merit of the reconstructed RGB full-color image using our three combinations, 4:2:0(A)-Ours $\times C_{u}$, relative to the above-mentioned six comparative combinations. The magnified subimage in Fig. 6(b) is decoupled from a wood wall region in Fig. 6(a). For QP = 48, after performing the 9 considered combinations on Fig. 6(b), the 9 reconstructed magnified subimages for Fig. 6(b) are shown in Figs. 6(c)-(k). From Figs. 6(c)-(k), we observe that our combination "4:2:0(A)-ours- $c_{u}$ "", $c_{u} \in C_{u}$, still has a better visual effect than "4:2:0(A)-LM- $c_{u}$ " and "4:2:0(A)LO- $c_{u}$ ", also indicating the visual effect merit of our luma modification method.

\section{2) The BD-rate merit}

The quality-bitrate tradeoff metric "BD-rate" [1] indicates the average bitrate reduction percentage under the same quality circumstance. When setting four QP intervals, namely $[4,20],[12,24],[20,32]$, and $[28,40]$, we adopt the BD-rate metric to demonstrate the quality-bitrate tradeoff merit of our luma modification method.

We take the five combinations in $C_{s} \times \mathrm{LM}-\mathrm{NN}$ [4] as the five baselines. Then, based on the four QP intervals, the BD-rates of our five combinations, namely $C_{s} \times$ Ours- $I N T E R_{C U B I C}$ and the ten comparative combinations, namely $C_{s} \times \mathrm{LM}-I N T E R_{C U B I C}[4]$ and $C_{s} \times \mathrm{LO}$ $I N T E R_{C U B I C}$ [11], are used to show the quality-bitrate tradeoff merit of our luma modification method "Ours". From Table 3, we observe that for each $c_{s} \in C_{s}$, our combination " $c_{s}$-Ours-INTER $R_{C U B I C \text { " has better BD-rate performance }}$ in boldface among the three considered combinations, indicating the BD-rate merit of our luma modification.

\section{CONCLUSION}

For $I^{R G B}$, we have presented the proposed BCI-based iterative luma modification method for the CSFLM scheme. First, we propose a new and more effective BCI-based pixeldistortion function to measure the SSE between the ground truth RGB full-color pixel and the estimated one at the server side. Next, we prove that the proposed pixel-distortion function is a convex function. Then, we transform the luma modification problem in CSFLM to an overdetermined system, and we apply the pseudo-inverse technique to obtain the initial luma modification solution. Finally, based on the convex property of the proposed pixel-distortion function, a BCI-based iterative luma modification method is proposed to better enhance the quality of the reconstructed RGB fullcolor image. Based on the five datasets, the comprehensive experimental results have demonstrated the quality enhancement and the quality-bitrate tradeoff merits of our luma modification method relative to the state-of-the-art methods [4], [11].

The future work is to deploy our BCI-based luma modification method in Zhu et al.'s DCT quantization and color cross-space distortion minimization-based image compression method [21], and deploy our method in the cholesky decomposition, chroma subsampling, and luma modificationbased color image compression method [20] for achieving better quality performance of the reconstructed images.

\section{ACKNOWLEDGMENT}

The authors appreciate the proofreading help of Ms. C. Harrington to improve the manuscript. 


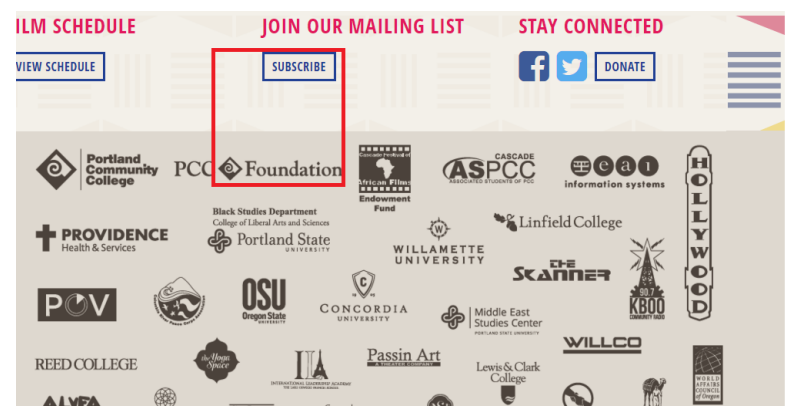

(a)

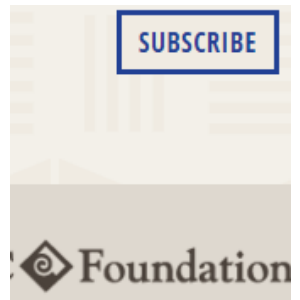

(b)

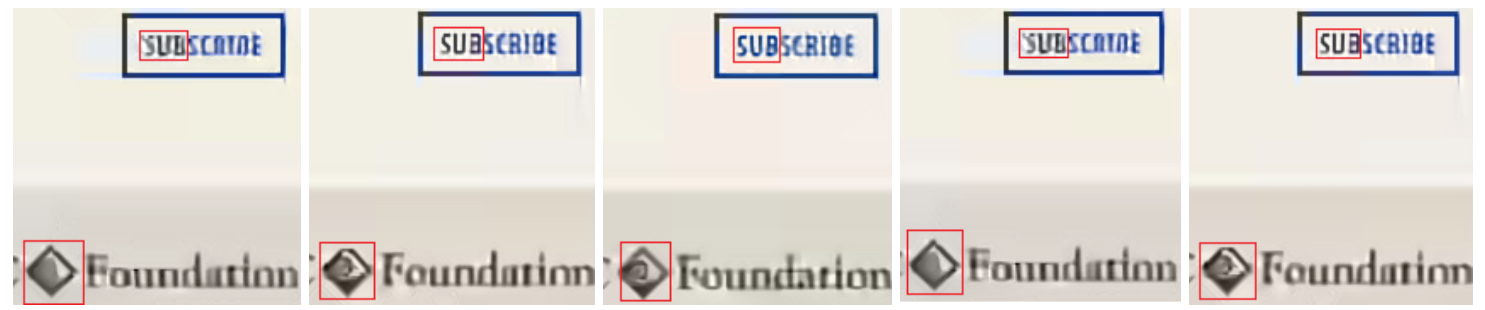

(c)

(d) (e) (f) (g)

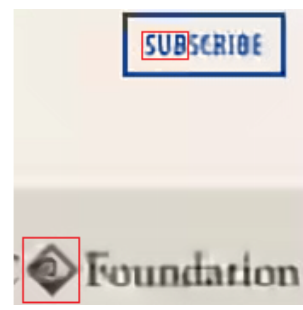

(h)

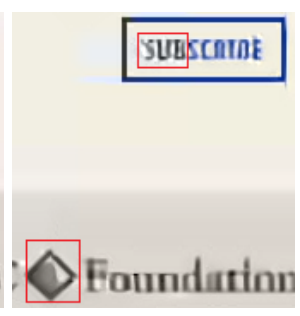

(i)

\section{SU⿴囗十⺝CBIBE}

SUBSCRIBE

FIGURE 5. The first visual effect merit example of our luma modification method. (a) The 5th ground truth $\mathrm{SCl}$ image. (b) The magnified subimage of (a). (c) 4:2:0(A)-LM-NN [4]. (d) 4:2:0(A)-LO-NN [11]. (e) 4:2:0(A)-Ours-NN. (f) 4:2:0(A)-LM-(4-tap). (g) 4:2:0(A)-LO-(4-tap) [1]. (h) 4:2:0(A)-Ours-(4-tap). (i) 4:2:0(A)-LM-INTER $R_{C U B I C}$ [4]. (j) 4:2:0(A)-LO-INTER $C U$ BIC [11]. (k) 4:2:0(A)-Ours-INTER $C U B I C$.

TABLE 2. FOR QP $=0$, THE QUALITY ENHANCEMENT MERIT OF OUR LUMA MODIFICATION METHOD "Ours" OVER THE LM METHOD [4] AND LO [1] METHODS.

\begin{tabular}{|c|c|c|c|c|}
\hline \multirow{2}{*}{ Combination } & \multicolumn{4}{|c|}{ Average } \\
\cline { 2 - 6 } & CPSNR & CPSNR gain & SSIM & SSIM gain \\
\hline 4:2:0(A)-LM-INTER $R_{C U B I C}$ & 41.8420 & & 0.9773 & \\
\hline $4: 2: 0(\mathrm{~A})-\mathrm{LO}-I N T E R_{C U B I C}$ & 41.8429 & 0.0009 & 0.9782 & 0.0009 \\
\hline $4: 2: 0(\mathrm{~A})-$ Ours- $I N T E R_{C U B I C}$ & $\mathbf{4 2 . 3 0 6 4}$ & $\mathbf{0 . 4 6 4 4}$ & $\mathbf{0 . 9 7 9 0}$ & $\mathbf{0 . 0 0 1 7}$ \\
\hline
\end{tabular}

\begin{tabular}{|c|c|c|c|c|}
\hline 4:2:0(L)-LM-INTE $R_{C U B I C}$ & 41.3268 & & 0.9751 & \\
\hline $4: 2: 0(\mathrm{~L})-\mathrm{LO}-I N T E R_{C U B I C}[\overline{1}$ & 41.3643 & 0.0375 & 0.9751 & 0 \\
\hline 4:2:0(L)-Ours-INTER $R_{C U B I C}$ & 42.1507 & 0.8239 & 0.9780 & 0.0029 \\
\hline $4: 2: 0(\mathrm{R})-\mathrm{LM}-I N T E R_{C U B I C}[4]$ & 41.1046 & & 0.9769 & \\
\hline $4: 2: 0(\mathrm{R})-\mathrm{LO}-I N T E R_{C U B I C}$ & 41.1652 & 0.0606 & 0.9749 & -0.0020 \\
\hline $4: 2: 0(\mathrm{R})$-Ours- $I N T E R_{C U B I C}$ & 42.0320 & 0.9274 & 0.9784 & 0.0015 \\
\hline
\end{tabular}

\begin{tabular}{|c|c|c|c|c|}
\hline 4:2:0(D)-LM- $I N T E R_{C U B I C}$ & 40.4921 & & 0.9712 & \\
\hline 4:2:0(D)-LO-INTER $R_{C U B I C}$ & 40.5725 & 0.0804 & 0.9712 & 0 \\
\hline 4:2:0(D)-Ours- $I N T E R_{C U B I C}$ & 41.6182 & 1.1261 & 0.9744 & $\mathbf{0 . 0 0 3 2}$ \\
\hline Anchor-LM- $I N T E R_{C U B I C}$ & 40.7387 & & 0.9761 & \\
\hline Anchor-LO-INTER $R_{C U B I C} \bar{\Pi}$ & 40.7939 & 0.0552 & 0.9762 & 0.0001 \\
\hline Anchor-Ours- $I N T E R_{C U B I C}$ & 41.3828 & 0.6441 & 0.9784 & 0.0023 \\
\hline
\end{tabular}




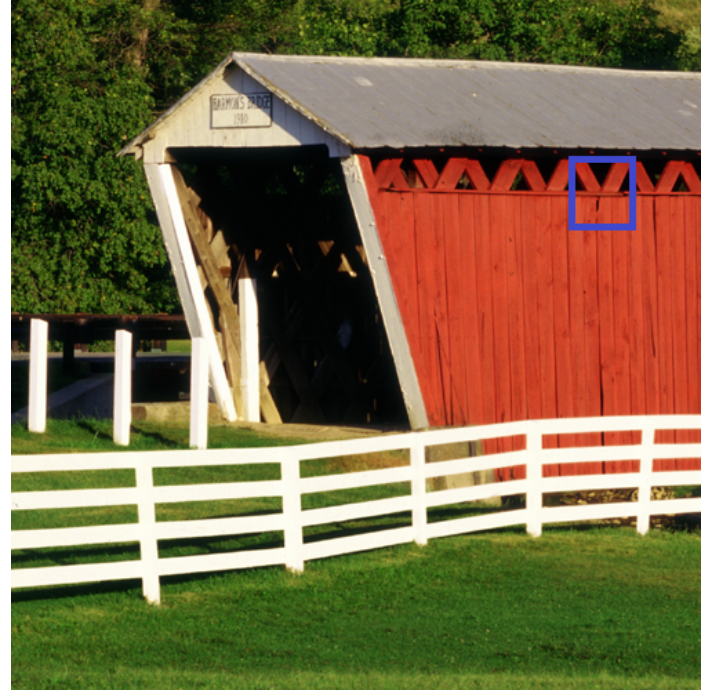

(a)

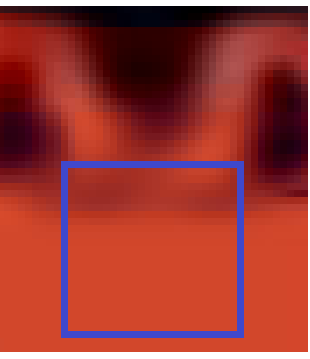

(c)

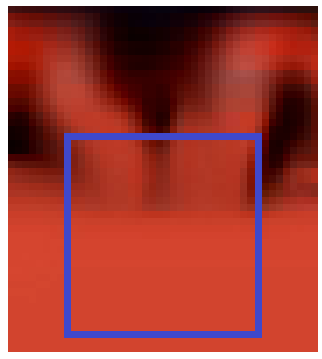

(d)

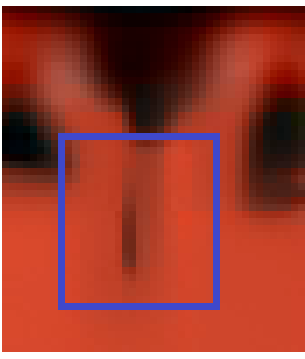

(e)

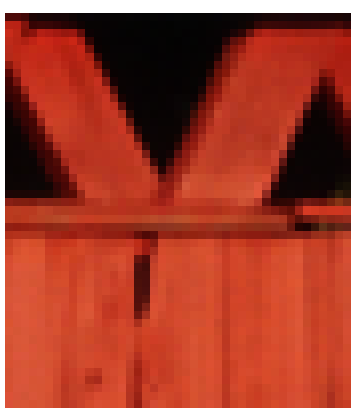

(b)

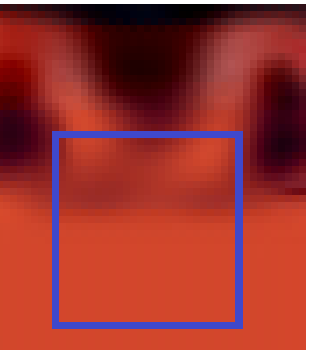

(f)

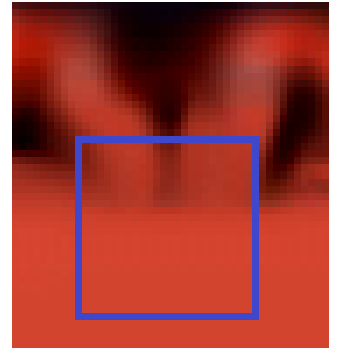

(g)

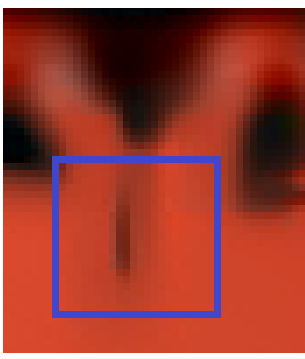

(h)

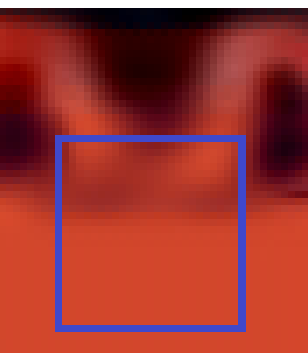

(i)

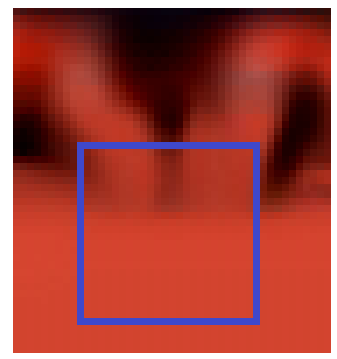

(j)

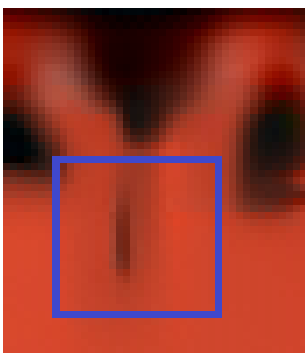

(k)

FIGURE 6. The second visual effect merit example of our luma modification method. (a) The 18th ground truth IMAX image. (b) The magnified subimage of (a). (c) 4:2:0(A)-LM-NN [4]. (d) 4:2:0(A)-LO-NN [11]. (e) 4:2:0(A)-Ours-NN. (f) 4:2:0(A)-LM-(4-tap). (g) 4:2:0(A)-LO-(4-tap) [1]. (h) 4:2:0(A)-Ours-(4-tap). (i)

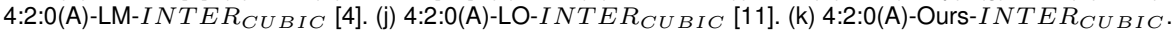

TABLE 3. AVERAGE BD-RATE (\%) MERIT OF OUR LUMA MODIFICATION METHOD RELATIVE TO THE LM METHOD [4] AND THE LO METHOD [1].

\begin{tabular}{|c|c|c|c|c|c|}
\hline BASELINE & & $\mathrm{QP}[4,20]$ & $\mathrm{QP}[12,24]$ & $\mathrm{QP}[20,32]$ & $\mathrm{QP}[28,40]$ \\
\hline \multirow{3}{*}{ 4:2:0(A)-LM-NN } & 4:2:0(A)-LM-INTER $R_{C U B I C}$ & -28.2011 & -19.4648 & -10.0312 & -4.9524 \\
\hline & $4: 2: 0(\mathrm{~A})-\mathrm{LO}-I N T E R_{C U B I C}$ & -26.3424 & -18.5149 & -9.8938 & -4.6914 \\
\hline & 4:2:0(A)-Ours-INTER $R_{C U B I C}$ & -35.2960 & -24.0350 & -12.0350 & -5.7430 \\
\hline \multirow{3}{*}{ 4:2:0(L)-LM-NN } & $4: 2: 0(\mathrm{~L})-\mathrm{LM}-I N T E R_{C U B I C}$ & -51.3053 & -33.2737 & -16.2604 & -8.0706 \\
\hline & $4: 2: 0(\mathrm{~L})-\mathrm{LO}-I N T E R_{C U B I C}$ & -50.4927 & -32.7949 & -16.7172 & -8.1473 \\
\hline & 4:2:0(L)-Ours- $I N T E R_{C U B I C}$ & -58.9572 & -42.6885 & -20.6550 & -10.4733 \\
\hline \multirow{3}{*}{ 4:2:0(R)-LM-NN } & 4:2:0(R)-LM- $I N T E R_{C U B I C}$ & -46.9029 & -30.8102 & -14.2375 & -6.3351 \\
\hline & 4:2:0(R)-LO-INTER $R_{C U B I C}$ & -46.5727 & -30.7609 & -15.1569 & -7.0160 \\
\hline & 4:2:0(R)-Ours- $I N T E R_{C U B I C}$ & -54.9912 & -39.4583 & -17.7729 & -8.0296 \\
\hline \multirow{3}{*}{ 4:2:0(D)-LM-NN } & 4:2:0(D)-LM- $I N T E R_{C U B I C}$ & -65.2660 & -46.0250 & -20.5181 & -9.5217 \\
\hline & 4:2:0(D)-LO-INTERCUBIC & -66.1487 & -46.6353 & -22.1845 & -10.9033 \\
\hline & 4:2:0(D)-Ours-INTER $R_{C U B I C}$ & -70.3740 & -58.5790 & -27.1803 & -13.5370 \\
\hline \multirow{3}{*}{ Anchor-LM-NN } & Anchor-LM-INTE $R_{C U B I C}$ & -48.2139 & -31.4733 & -15.2844 & -7.5494 \\
\hline & Anchor-LO-INTER $R_{C U B I C}$ & -48.2022 & -31.8881 & -16.1559 & -7.8624 \\
\hline & Anchor-Ours- $I N T E R_{C U B I C}$ & -56.2545 & -39.7256 & -19.3293 & -9.5309 \\
\hline
\end{tabular}




\section{REFERENCES}

[1] G. Bjøntegaard, Calculation of average PSNR difference between RDcurves, ITU-T SG16/Q6 VCEG, Austin, TX, USA, document VECGM33, pp. 2-4, Apr. 2001

[2] S. Boyd and L. Vandenberghe, Convex Optimization, Cambridge, U.K.: Cambridge Univ. pp. 67, 2004.

[3] G. Bradski and A. Kaehler, Learning OpenCV: Comput. Vision with the OpenCV Library. Sebastopol, CA, USA: O’Reilly Media, 2008.

[4] K. L. Chung, T. C. Hsu, and C. C. Huang, "Joint chroma subsampling and distortion-minimization-based luma modification for RGB color images with application," IEEE Transactions on Image Processing, vol. 26, no. 10, pp. 4626-4638, Oct. 2017.

[5] Execution code of the our BCI-based iterative luma modifi-cation method. [Online]. Available: Ftp://140.118.175.164/CSFLM/CSFLM.zip.

[6] HM-16.18. accessed on Jan. 2018. [Online]. Available: https://hevc.hhi. fraunhofer.de/svn/svn_HEVCSoftware/tags/HM-16.18/

[7] IMAX true color image collection, 2014. [Online]. Available https:// www4.comp.polyu.edu.hk/ cslzhang/CDM_Dataset.htm

[8] ITU-R Recommendation BT-601-5: Studio encoding parameters of digital television for standard 4:3 and wide-screen 16:9 aspect ratios. International Telecommunications Union, 2011.

[9] R. G. Keys, "Cubic convolution interpolation for digital image processing," IEEE transactions on acoustics, speech, and signal processing, vol. 29, no. 6, pp. 1153-1160, Jan. 28, Dec. 1981.

[10] Kodak true color image collection, 2014. [Online]. Available: https://www. math.purdue.edu/ lucier/PHOTO_CD/BMP_IMAGES/

[11] T. L. Lin, B. H. Liu, and K. H. Jiang, "An efficient algorithm for luminance optimization in chroma downsampling," IEEE Transactions on Circuits and Systems for Video Technology, Early Access, 2021.

[12] A. Luthra, E. Franc, ois, and W. Husak, Call for evidence (CfE) for HDR and WCG video coding, Standard ISO/IEC JTC1/SC29/WG11 (MPEG), Geneva, Switzerland, Feb. 2015.

[13] Open CV codes. [Online]. Available: https:// sourceforge.net/projects/opencvlibrary/?fbclid=IwAR2vXid ZecTmGKWiCuOe2XuaCKdAq4-Aes7FfeEVIpDM-BwOJAgg6TbI-k

[14] SCI image database, accessed on Jan. 10, 2016. [Online]. Available: ftp: //140.118.175.164/SCI

[15] Spatial Scalability Filters, document ISO/IEC JTC1/SC29/WG11 ITU-T SG 16 Q.6, Jul. 2005.

[16] M. Tachi, "Image processing device, image processing method, and program pertaining to image correction," U.S. Patent 8,314,863, Nov. 20, 2012.

[17] The video dataset. [Online]. Available: ftp://140.118.175.164/CFASS/

[18] VTM-11.0, 2021. [Online]. Available: https://vcgit.hhi.fraunhofer.de/jvet/ /VVCSoftware_VTM

[19] Z. Wang, A. C. Bovik, H. R. Sheikh, E. P. Simoncelli, "Image quality assessment: from error visibility to structural similarity," IEEE Transactions on Image Processing, vol. 13, no. 4, pp. 600-612, Apr. 2004.

[20] S. Zhu, C. Cui, R. Xiong, Y. Guo, and B. Zeng, "Efficient chroma subsampling and luma modification for color image compression," IEEE Transactions on Circuits and Systems for Video Technology, vol. 29, no. 5, pp. 1559-1563, May. 2019.

[21] S. Zhu, M. Li, C. Chen, S. Liu, and B. Zeng, "Cross-space distortion directed color image compression," IEEE Transactions on Multimedia, vol. 20, no. 3, pp. 525-538, 2018, Mar. 2018

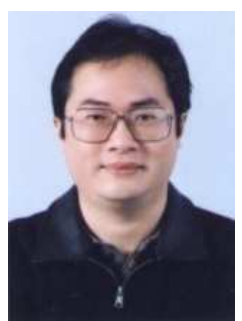

KUO-LIANG CHUNG (SM01)received his B.S., M.S., and Ph.D. degrees from National Taiwan University, Taipei, Taiwan in 1982, 1984, and 1990, respectively. He has been one Chair Professor of the Department of Computer Science and Information Engineering at National Taiwan University of Science and Technology, Taipei, Taiwan since 2009. He was the recipient of the Distinguished Research Award (2004-2007; 20192022) and Distinguished Research Project Award (2009-2012) from the Ministry of Science and Technology of Taiwan. He has been an Associate Editor of the Journal of Visual Communication and Image Representation since 2011. His research interests include image processing, video compression, and deep learning.

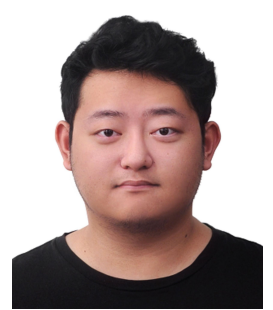

CHIH-YUAN HUANG received his B.S. degree in Computer Science and Engineering from the National Changhua University of Education, Changhua, Taiwan, in 2019. He is currently working towards his M.S. degree in Computer Science and Information Engineering at the National Taiwan University of Science and Technology, Taipei, Taiwan. His research interests include image processing and video compression.

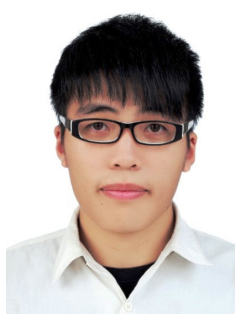

CHEN-WEI KAO received his B.S. degree in Computer Science and Engineering from the National Taiwan Ocean University, Keelung, Taiwan, in 2019. He received his M.S. degree in Computer Science and Information Engineering at the $\mathrm{Na}$ tional Taiwan University of Science and Technology, Taipei, Taiwan, in 2021. His research interests include image processing and video compression. 